\title{
CORRECTIONS
}

\section{ON APPLICATIONS OF EXCESS LEVEL PROCESSES TO (N,D)-POLICY BULK QUEUEING SYSTEMS}

\author{
JEWGENI H. DSHALALOW \\ Department of Applied Mathematics \\ Florida Institute of Technology \\ Melbourne, FL 32901, U.S.A. \\ e-mail: eugene@winnie.FIT.edu
}

In the above paper, published in the Journal of Applied Mathematics and Stochastic Analysis [1], several formulas and phrases are found to be erroneous. Although the listed below corrections fix the problems, the full corrected version reads better and is now available as a technical report [2].

Correction 1. Page 553, lines 13-19 should become:

$\ldots$ such that for each $n,\left(X_{n}, Y_{n}\right)$ depend only on the $n$th interenewal time $\tau_{n}-\tau_{n-1}$. Consequently,

$$
(A, B, \tau)=\left\{\left(A_{n}=\sum_{k=0}^{n} X_{k}, B_{n}=\sum_{k=0}^{n} Y_{k}, \tau_{n}\right) ; n=0,1, \ldots\right\}
$$

is a three-dimensional delayed renewal process.

Process $Z$ will be described in terms of the following transformations:

$$
\begin{gathered}
\gamma_{0}(z, \vartheta, \theta)=\mathbb{E}\left[z^{X_{0}} e^{-\vartheta Y_{0}} e^{-\theta \tau_{0}}\right], \gamma(z, \vartheta, \theta)=\mathbb{E}\left[z^{X_{1}} e^{-\vartheta Y_{1}} e^{-\theta\left(\tau_{1}-\tau_{0}\right)}\right], \\
|z| \leq 1, \operatorname{Re}(\vartheta) \geq 0, \operatorname{Re}(\theta) \geq 0, \\
\alpha_{0}(z)=\gamma_{0}(z, 0,0), \alpha(z)=\gamma(z, 0,0), \\
\gamma_{0}(z, \vartheta)=\gamma_{0}(z, \vartheta, 0), h_{0}(\theta)=\gamma_{0}(1,0, \theta), \gamma(z, \vartheta)=\gamma(z, \vartheta, 0), h(\theta)=\gamma(1,0, \theta),
\end{gathered}
$$

Correction 2. Theorem 1 on page 554, lines 14-15, should be restated as follows:

Theorem 1. Let $\left(X_{n}, Y_{n}\right)$ be independent of $\tau_{n}-\tau_{n-1}$ for each $n$. Then,

$$
\frac{1}{h_{0}(\theta)} \mathcal{F}^{*}(\xi, \theta, z, \vartheta ; x, s)=\gamma_{0}(z, \vartheta)-\gamma_{0}(x z, \vartheta+s) \frac{1-\xi h(\theta) \gamma(z, \vartheta)}{1-\xi h(\theta) \gamma(x z, \vartheta+s)}
$$

Insert 3. Following Theorem 1, we add Theorem 1a:

Theorem 1a. With no above assumption on the independence,

$$
\mathscr{F}^{*}(\xi, \theta, z, \vartheta ; x, s)=\gamma_{0}(z, \vartheta, \theta)-\gamma_{0}(x z, \vartheta+s, \theta) \frac{1-\xi \gamma(z, \vartheta, \theta)}{1-\xi \gamma(x z, \vartheta+s, \theta)}
$$


208 CORRECTIONS to On Applications of Excess Level ..., by J.H. Dshalalow

Correction 4. Page 559, lines 10-12 should read:

$$
\begin{aligned}
& \gamma(z, \vartheta, \theta)=\mathbb{E}\left[z^{X_{1}^{*}} e^{-\vartheta Y_{1}^{*}} e^{-\theta\left(\tau_{1}-\tau_{0}\right)}\right] \\
& =\mathbb{E}\left[\mathbb{E}\left[\left[\mathbb{E}\left[z^{X_{1}^{*}} e^{-\vartheta Y_{1}^{*}} e^{-\theta\left(\tau_{1}-\tau_{0}\right)}\right] \mid X_{1}^{*}\right] \mid \tau_{1}-\tau_{0}\right]\right. \\
& =\varphi\{\theta+\lambda[1-\alpha(z \beta(\vartheta))]\} .
\end{aligned}
$$

The three-dimensional benchmark process, to which the busy period policy is applied, is now

$$
(A, B, \tau)=\left\{\left(A_{n}=\sum_{k=0}^{n} X_{k}^{*}, B_{n}=\sum_{k=0}^{n} Y_{k}^{*}, \tau_{n}\right) ; n=0,1, \ldots\right\} .
$$

Correction 5. Page 559, line 16 should be corrected as

$\ldots$ the marginal transformation $\gamma(z, 0,0)$ satisfies formula $(6.1)$.

Correction 6. Page 559, lines 18-19 should read:

$$
\begin{aligned}
& \mathbb{E}^{0}\left[\xi^{\nu} e^{\left.-\theta r_{\nu}{ }^{A} \nu\right]}\right. \\
& =1-[1-\xi \varphi\{\theta+\lambda(1-\alpha(z))\}] \mathcal{L}_{D} \mathscr{D}_{x}^{N-1}\left\{\frac{1}{1-\xi \varphi\{\theta+\lambda(1-\alpha(x z \beta(s))\}}\right\}
\end{aligned}
$$

\section{References}

[1] Dshalalow J.H., On applications of excess level processes to (N,D)-policy bulk queueing systems, JAMSA, 9:4 (1996), 551-562.

[2] Dshalalow J.H., On applications of excess level processes to (N,D)-policy bulk queueing systems, Tech. Report, MS-19702 (1997), 1-12. 


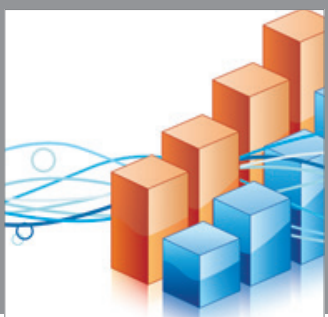

Advances in

Operations Research

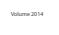

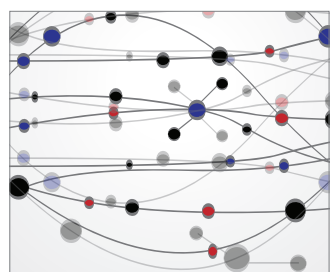

\section{The Scientific} World Journal
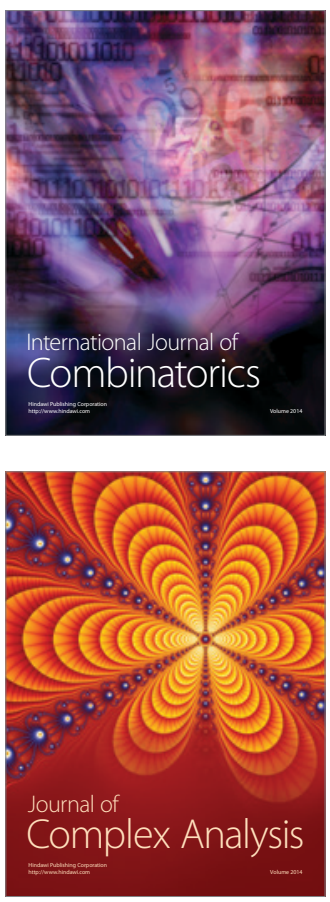

International Journal of

Mathematics and

Mathematical

Sciences
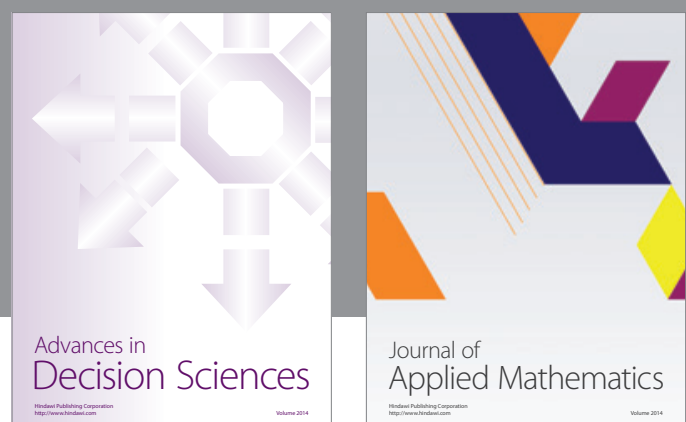

Journal of

Applied Mathematics
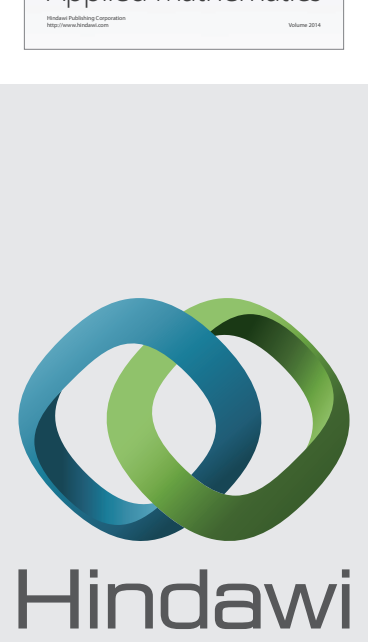

Submit your manuscripts at http://www.hindawi.com
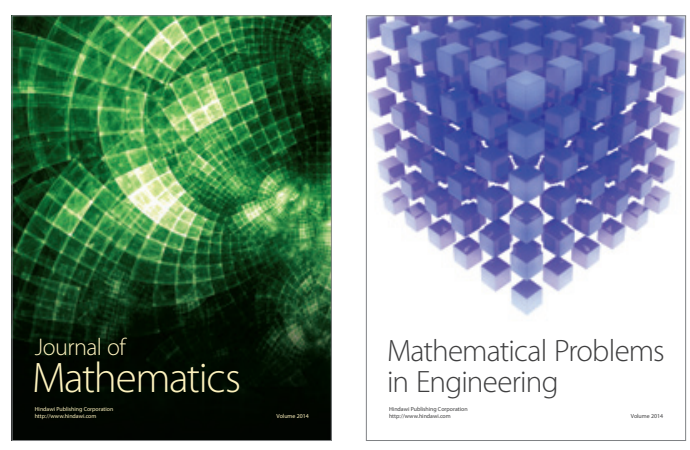

Mathematical Problems in Engineering
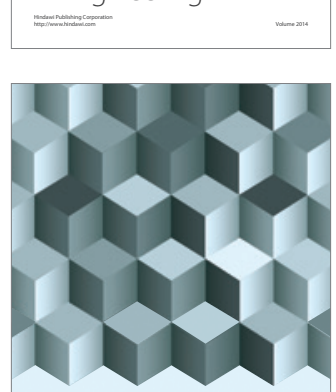

Journal of

Function Spaces
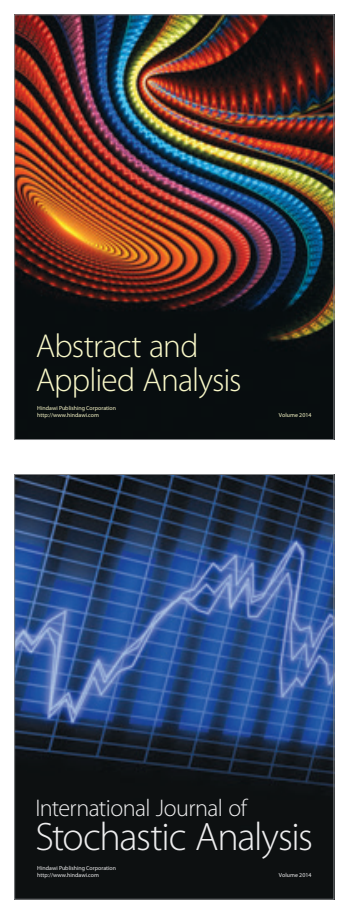

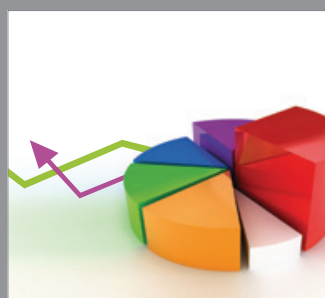

ournal of

Probability and Statistics

Promensencen
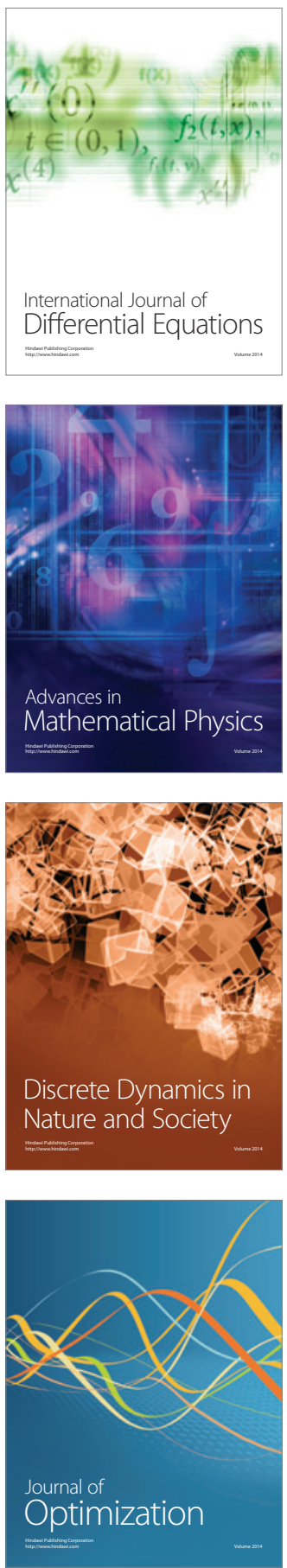